NASA/TM-2005-213654

\title{
A Robust Absorbing Boundary Condition for Compressible Flows
}

Ching Y. Loh

Taitech, Inc., Beavercreek, Ohio

Philip C.E. Jorgenson

Glenn Research Center, Cleveland, Ohio 
Since its founding, NASA has been dedicated to the advancement of aeronautics and space science. The NASA Scientific and Technical Information (STI) Program Office plays a key part in helping NASA maintain this important role.

The NASA STI Program Office is operated by Langley Research Center, the Lead Center for NASA's scientific and technical information. The NASA STI Program Office provides access to the NASA STI Database, the largest collection of aeronautical and space science STI in the world. The Program Office is also NASA's institutional mechanism for disseminating the results of its research and development activities. These results are published by NASA in the NASA STI Report Series, which includes the following report types:

- $\quad$ TECHNICAL PUBLICATION. Reports of completed research or a major significant phase of research that present the results of NASA programs and include extensive data or theoretical analysis. Includes compilations of significant scientific and technical data and information deemed to be of continuing reference value. NASA's counterpart of peerreviewed formal professional papers but has less stringent limitations on manuscript length and extent of graphic presentations.

- TECHNICAL MEMORANDUM. Scientific and technical findings that are preliminary or of specialized interest, e.g., quick release reports, working papers, and bibliographies that contain minimal annotation. Does not contain extensive analysis.

- CONTRACTOR REPORT. Scientific and technical findings by NASA-sponsored contractors and grantees.
- CONFERENCE PUBLICATION. Collected papers from scientific and technical conferences, symposia, seminars, or other meetings sponsored or cosponsored by NASA.

- SPECIAL PUBLICATION. Scientific, technical, or historical information from NASA programs, projects, and missions, often concerned with subjects having substantial public interest.

- TECHNICAL TRANSLATION. Englishlanguage translations of foreign scientific and technical material pertinent to NASA's mission.

Specialized services that complement the STI Program Office's diverse offerings include creating custom thesauri, building customized databases, organizing and publishing research results ... even providing videos.

For more information about the NASA STI Program Office, see the following:

- Access the NASA STI Program Home Page at http://www.sti.nasa.gov

- E-mail your question via the Internet to help@sti.nasa.gov

- Fax your question to the NASA Access Help Desk at 301-621-0134

- Telephone the NASA Access Help Desk at 301-621-0390

- Write to:

NASA Access Help Desk

NASA Center for AeroSpace Information 7121 Standard Drive

Hanover, MD 21076 
NASA/TM-2005-213654

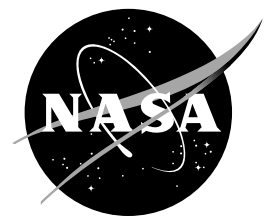

\section{A Robust Absorbing Boundary Condition for Compressible Flows}

Ching Y. Loh

Taitech, Inc., Beavercreek, Ohio

Philip C.E. Jorgenson

Glenn Research Center, Cleveland, Ohio

Prepared for the

17th Computational Fluid Dynamics Conference

sponsored by the American Institute of Aeronautics and Astronautics

Toronto, Ontario, Canada, June 6-9, 2005

National Aeronautics and

Space Administration

Glenn Research Center 


\section{Acknowledgments}

This work received support from the Quiet Aircraft Technology Project Office of NASA Glenn Research Center.

This report contains preliminary

findings, subject to revision as analysis proceeds.

Available from

NASA Center for Aerospace Information 7121 Standard Drive

Hanover, MD 21076
National Technical Information Service 5285 Port Royal Road Springfield, VA 22100 


\title{
A Robust Absorbing Boundary Condition for Compressible Flows
}

\author{
Ching Y. Loh \\ Taitech Inc. \\ Beavercreek, Ohio 45430 \\ Philip C.E. Jorgenson \\ National Aeronautics and Space Administration \\ Glenn Research Center \\ Cleveland, Ohio 44135
}

\begin{abstract}
An absorbing non-reflecting boundary condition (NRBC) for practical computations in fluid dynamics and aeroacoustics is presented with theoretical proof. This paper is a continuation and improvement of a previous paper [2] by the author. The absorbing NRBC technique is based on a first principle of nonreflecting, which contains the essential physics that a plane wave solution [1] of the Euler equations remains intact across the boundary.

The technique is theoretically shown to work for a large class of finite volume approaches. When combined with the hyperbolic conservation laws, the NRBC is simple, robust and truly multi-dimensional; no additional implementation is needed except the prescribed physical boundary conditions. Several numerical examples in multi-dimensional spaces using two different finite volume schemes are illustrated to demonstrate its robustness in practical computations. Limitations and remedies of the technique are also discussed.
\end{abstract}

\section{Introduction}

It is well-known that non-reflecting boundary conditions (NRBCs) play a key role in fluid dynamics and aeroacoustics computations and still remain an important topic in the current research areas. A spurious reflection resulting from an inappropriate numerical boundary condition contaminates the flow field and may eventually spoil the entire flow computation. Research on NRBC is a challenging topic in engineering and applied mathematics. A vast number of papers on the topic of NRBCs have been published in past decades to treat various computational problems. Details can be found in the recent reviews [3, 4, 5] and the references cited therein.

Generally there are two types of non-reflecting boundaries (see e.g., [3]). One (Type I) is the true domain boundary where physical boundary conditions are specified and the other (Type II) is the artificially imposed boundary.

In this paper, we focus only on the Type I absorbing non-reflecting boundary. For a true boundary where the actual physical boundary conditions are given as per the physical problem, the NRBC needs to play a dual role: on the one hand it must be consistent with the given physical boundary conditions, on the other hand, it must be able to absorb waves or disturbances from the domain interior.

For the absorbing NRBC, there are two ways to absorb the outgoing waves/disturbances: the 'hard absorbing' and the 'soft absorbing'. In the 'hard absorbing', the given boundary condition must be modified to an admissible one. This can be done using the characteristics method. In one-dimensional flow, at the boundary, Enquist and Majda [6], and Hedstrom [7] showed that a boundary condition considered to be non-reflecting is equivalent to saying that the characteristic variables corresponding to the incoming characteristic curve remain unchanged across the boundary (see 
also Hirsch [8], p. 370). When the flow is subsonic, Riemann invariants (one-dimensional characteristic variables) are used to turn a prescribed boundary condition into an admissible NRBC. For multi-dimensional flow, this onedimensional technique is combined with dimension splitting. Such a combined treatment has been the topic of many papers (see e.g. [9] and references in [3]). The characteristics based NRBC is the most popular numerical treatment, despite its complicated implementation.

The 'soft absorbing' treatments found in the literature generally include (but not limited to):

(i)A buffer zone (sponge zone) technique can be used to add a non-physical zone between the domain core and the boundary to diminish the strength of the outgoing waves/disturbances before they reach the boundary, thus minimizing the reflecting effect. In the buffer zone, either artificially increased dissipation is applied or grid stretching is imposed (see e.g. [10,18]), which is equivalent to numerical filtering.

(ii) In the recently developed perfectly matched layer (PML) method $[11,12]$, a specially designed equation system is imposed in the matching layer (or absorbing layer) at the boundary to guarantee the exponential decay of the disturbances in the layer.

In addition to the existing absorbing NRBCs, as mentioned in [2], a new absorbing (Type I) NRBC technique was recently discovered in an empirical way. The technique is robust but simple to implement and has been successfully employed in many computations $[16,17,18]$.

It is the purpose of the present paper to revisit the basic theoretical background of the absorbing boundary condition and to explain why the technique works well for flows in one to three dimensional spaces. Starting from a first principle of non-reflection, i.e., there is no reflection at any interior point of a computational domain, a $C^{1}$ continuity criterion for flow variables is introduced as a generic NRBC. The criterion is then incorporated in the time-dependent hyperbolic conservation laws in discretized form, and the consequent absorbing (Type I) NRBC technique is described and theoretically proven.

In the present paper, we first develop the basic principle and criterion for the continuous problem and then apply them to the discretized numerical problems. The paper is arranged as follows: in $\S 2$, based on a first principle of non-reflection, the $C^{1}$ continuity criterion of NRBC is introduced and proven. Based on this continuity criterion, the absorbing NRBC is depicted in $\S 3$. Various numerical examples in two or three-dimensional spaces with two different finite volume schemes are illustrated to demonstrate the capability of the new NRBC. Limitations and remedies of the absorbing NRBC are discussed in $\S 5$. Finally, the paper is concluded with remarks in $\S 6$.

Throughout the present paper,

$$
\mathbf{V}=\left(v_{1}, v_{2}, v_{3}, v_{4}, v_{5}\right)^{T}=(\rho, u, v, w, p)^{T}
$$

is employed to represent the primitive flow variables (for notations, see below). The primitive flow variables $\mathbf{V}$ and the original non-conservative form of the Euler equations are conveniently employed in the theoretical investigation but hyperbolic conservation laws in discretized form are needed in the numerical implementation. Here is the standard three-dimensional conservation form of the non-dimensional Euler equations:

$$
\boldsymbol{U}_{t}+\boldsymbol{F}_{x}+\boldsymbol{G}_{y}+\boldsymbol{H}_{z}=\mathbf{Q},
$$

where $x, y, z$ and $t$ are the streamwise and transverse coordinates and time, respectively. The conservative flow variable vector, $\boldsymbol{U}$, and the flux vectors, $\boldsymbol{F}, \boldsymbol{G}, \boldsymbol{H}$, are given by:

$$
\boldsymbol{U}=\left(\begin{array}{c}
U_{1} \\
U_{2} \\
U_{3} \\
U_{4} \\
U_{5}
\end{array}\right)=\left(\begin{array}{c}
\rho \\
\rho u \\
\rho v \\
\rho w \\
\rho e
\end{array}\right), \boldsymbol{F}=\left(\begin{array}{c}
\rho u \\
\rho u^{2}+p \\
\rho u v \\
\rho u w \\
\rho u H
\end{array}\right), \quad \boldsymbol{G}=\left(\begin{array}{c}
\rho v \\
\rho u v \\
\rho v^{2}+p \\
\rho v w \\
\rho v H
\end{array}\right), \boldsymbol{H}=\left(\begin{array}{c}
\rho w \\
\rho w u \\
\rho w v \\
\rho w^{2}+p \\
\rho w H
\end{array}\right) .
$$

where $u, v, w$ and $\rho, p$ are respectively the velocity components, density and pressure, with the internal energy $e=$ $\frac{p}{\rho(\gamma-1)}+1 / 2\left(u^{2}+v^{2}+w^{2}\right)$, the enthalpy $H=p / \rho+e$, and $\gamma=1.4$. The right hand side $\mathbf{Q}$ is the source term which may possibly include the external forcing terms.

By considering $(x, y, z, t)$ as coordinates of a four-dimensional Euclidean space, $E_{4}$, and using the Gauss divergence theorem, it follows that Eq. (1) is equivalent to the following integral form of the conservation laws:

$$
\oint_{S(V)} \mathbf{I}_{\mathbf{m}} \cdot \mathrm{d} \mathbf{s}=\int_{V} \mathbf{Q}_{\mathbf{m}} \mathrm{d} V, \quad m=1,2,3,4,5,
$$


where $S(V)$ denotes the surface around a control volume, $V$, in $E_{4}$ and $\mathbf{I}_{\mathbf{m}}=\left(F_{m}, G_{m}, H_{m}, U_{m}\right)$ represents the flux vectors. The vector differential $\mathbf{d} \mathbf{s}=\mathbf{n} d s$, where $\mathbf{n}$ is the outgoing unit normal vector of the infinitesimal surface element $d s$ in $E_{4}$.

\section{A first principle of non-reflection and the continuity criterion}

In this section, a theoretical analysis of non-reflecting is conducted for the continuous problem (as opposed to a discretized problem) of the Euler equations. Then, it is applied to the discretized NRBCs in the sections that follow. For clarity, the flow variables are assumed to be continuous near the boundary, and all the spatial and temporal derivatives exist. It should be stated that, even though discontinuities such as shocks or contacts may develop and propagate across the boundary, for a discretized absorbing NRBC, such continuous flow requirement is always satisfied locally at the boundary via the numerical procedure, and hence the following first principle and criterion of non-reflecting are still valid.

From the classical theory (e.g. Courant and Hilbert[1]) of partial differential equation (PDE), it is well known that hyperbolic PDE systems support wave solutions. In the present paper, we specifically focus on the Euler equations. Due to their hyperbolicity, they support linear continuous waves such as pressure pulse waves, entropy (density) waves, and vorticity waves as well as nonlinear or discontinuous waves such as shocks.

\subsection{A first principle of non-reflection}

As the PDE system supports wave solutions, the next issue is focussed on the questions: what is 'non-reflecting' and how a boundary can become non-reflecting? Hereby, instead of following the conventional wisdom of a signal/wave propagating along characteristics, the concept of 'non-reflecting' is viewed from a different but simple perspective.

Recall a general observation that, no matter how crude the numerical scheme is, spurious reflection can only be generated at the domain boundaries, no reflection ever occurs at any interior point. This is because only at the boundary is the numerical solution subjected to two competing conditions, namely, the governing equations and the imposed boundary conditons.

The following logical argument further justifies that no reflection should occur in the domain interior: At an interior point of the domain, the solution is subjeted only to the governing equations. For any scheme that converges, the numerical result at this point, which may even contain the possible spurious reflection propagated from a boundary, is literally considered to be $100 \%$ a discretized solution of the governing equations and nothing else, disregarding any discretization error. Conceptually, no such 'impurity' as 'reflection' is generated at this interior point.

Theoretically, for a hyperbolic PDE system, locally in a small neighborhood of any interior point $O$, by using Cauchy's method of the Fourier integral (e.g.,[1], p.210, or [ 8 ]), the homogeneous part of the solution of the initial problem, which may contain oscillations, can be expressed by the superposition of plane waves:

$$
\mathbf{V}=\sum_{m=1}^{M} \int_{-\infty}^{\infty} \ldots \int_{-\infty}^{\infty} \tilde{\mathbf{V}}_{m}(\mathbf{k}) e^{i \theta} d k_{1} d k_{2} \ldots d k_{n},
$$

Here, $M$ is the number of PDE equations in the system, $\mathbf{k}=\left(k_{1}, k_{2}, \ldots, k_{n}\right)$ is the $n$-dimensional wave number vector, with $k_{j}, j=1,2, \ldots, n$, ranging from $-\infty$ to $\infty, i=\sqrt{-1}$, and $\mathbf{x}=\left(x_{1}, x_{2}, \ldots, x_{n}\right)$ is the $n$-dimensional position vector of this point $O . \tilde{\mathbf{V}}_{m}=\tilde{\mathbf{V}}_{m}(\mathbf{k})$ and $\theta=\theta(\mathbf{k}, \mathbf{x}, t)=\mathbf{x} \bullet \mathbf{k}-\omega_{m} t$ are respectively the amplitude and the phase of the plane wave, where $\omega_{m}=\omega_{m}(\mathbf{k})$ (a real number) is the angular frequency and can be determined as a function of the wave number vector $\mathbf{k}$ by substituting the single plane wave $\tilde{\mathbf{V}} e^{i \theta}$ into the PDE system. For each plane wave component in (3), as its amplitude and phase are functions of $\mathbf{k}$ and remain intact when passing through this interior point $O$, no distortion or reflection occurs. So also for the solution $\mathbf{V}$.

Based on such observation, logical reasoning, and the theory of hyperbolic PDE system, we conclude that if all the points at the boundary are actually interior points of the domain, there is no reflection. A first principle of non-reflection is then proposed and adopted in the present paper:

A boundary of the solution domain is said to be non-reflecting if every point on the boundary surface can be considered as an interior point.

There are two requirements defining an interior point: 


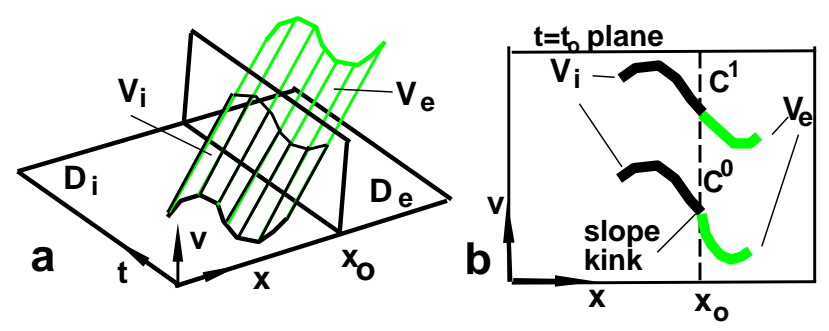

Figure 1. a: Sketch of a one-dimensional scalar plane wave, $b$ : the curves $v=v(x, t)$ on a plane $t=t_{0}$ at the boundary $x=x_{0}$, showing intuitively that $C^{1}$ continuity is necessary for non-reflection.

1. the point must be physically located within the domain;

2. the consistency requirement that the governing PDE system is the only condition to be satisfied at this point.

Other conditions, if any, must be equivalent to or consistent with the PDE system.

In order that all the points along the boundary be interior points, of course, the solution domain must be extended to include at least a small neighborhood around the boundary. Numerically, the ghost cell is a natural choice for the neighborhood at the boundary. The consistency requirement is a more important issue. An NRBC is sought such that it fully complies with the governing equations, that is, solutions in the domain interior and in the domain exterior must be mutually a solution continuation of each other, satisfying the same PDE system in the extended domain.

This concept or definition of non-reflection leads naturally to a generic NRBC - the continuity criterion for Euler equations, which will be proposed and proven in $\$ 2.2$. The definition is a general concept and may be applied to hyperbolic systems other than the Euler equations.

\subsection{The continuity criterion - a generic NRBC}

With the presence of the boundary ( a cylindrical hyper-surface), the four-dimensional Euclidean time-space $E_{4}$ is bisected into two portions, domain interior $D_{i}$ and domain exterior $D_{e}$ (Figure 1a illustrates a 1-D scalar plane wave in $E_{2}$, only the real component of $\mathbf{V}$ is shown. The subscripts $i$ and $e$ stand respectively for domain interior and domain exterior ). Within $D_{i}$, the flow is governed by the original Euler equation system. It is also assumed that in the domain exterior $D_{e}$, at least in a neighborhood of the boundary, the flow is still governed by an Euler equation system. If the two Euler equation systems are identical at all the points of the boundary, the consistency requirement is satisfied, and these points are interior points of the extended domain. The boundary is hence non-reflective.

The continuity criterion leads naturally to such non-reflection, it is stated as follows:

At each point of the boundary, the flow variables $\mathbf{V}$ and their spatial derivatives, namely, $\mathbf{V}_{x}, \mathbf{V}_{y}, \mathbf{V}_{z}$, are continuous; or, $\mathbf{V}$ is $C^{1}$ continuous in space across the boundary.

It can be easily shown that locally there is no reflection at the boundary surface if the continuity criterion is satisfied.

Proof: Consider the non-conservation form of the Euler equations ( e.g., see [8]) for both domain interior and exterior:

$$
\frac{\partial \mathbf{V}}{\partial t}+\tilde{A} \frac{\partial \mathbf{V}}{\partial x}+\tilde{B} \frac{\partial \mathbf{V}}{\partial y}+\tilde{C} \frac{\partial \mathbf{V}}{\partial z}=\tilde{\mathbf{Q}} .
$$

Here $\tilde{\mathbf{Q}}$ is a nonhomogeneous source term vector and may be a vector function of $x, y, z, t$ and $\mathbf{V}$, and could include forcing terms.

Note that the jacobian matrices $\tilde{A}, \tilde{B}$, and $\tilde{C}$ are directly functions of $\mathbf{V}$ (details may be found in [8]). As a result of the $C^{1}$ spatial continuity of $\mathbf{V}$, all the terms (items)

$$
\frac{\partial \mathbf{V}}{\partial t}, \quad \frac{\partial \mathbf{V}}{\partial x}, \quad \frac{\partial \mathbf{V}}{\partial y}, \quad \frac{\partial \mathbf{V}}{\partial z}, \quad \tilde{A}, \tilde{B}, \tilde{C}, \quad \text { and } \quad \tilde{\mathbf{Q}}
$$

in the governing Euler equations are identical across the boundary surface. Continuity of $\frac{\partial \mathbf{V}}{\partial t}$ is directly inferred from (4). Now, (4) can be considered as a single governing equation system in an extended domain which covers the boundary as its interior. The consistency requirement is satisfied at all the boundary points; hence, there is no reflection at the boundary. 


\subsection{Physical interpretation of the continuity criterion}

For the three-dimensional Euler equations, corresponding to Eqn. (3), $M=5$ and $n=3$. As a special case of the hyperbolic PDE system in $\S 2.1$, locally in a small neighborhood of an interior point, the homogeneous solution of the Euler equations can always be decomposed into plane waves having a vector form [8]:

$$
\mathbf{V}=\tilde{\mathbf{V}} e^{i \theta}
$$

Here, as in $\S 2.1, \tilde{\mathbf{V}}=\tilde{\mathbf{V}}(\mathbf{k}, x, y, z)$ is the 'amplitude', and $\theta=\theta(\mathbf{x}, t)=\mathbf{x} \bullet \mathbf{k}-\omega t$ is the phase of the plane wave, where $\mathbf{k}=\left(k_{x}, k_{y}, k_{z}\right)$ is the given wave number vector and $\omega=\omega(\mathbf{k})$ (a real number) is the angular frequency. and $\mathbf{x}=(x, y, z)$ the position vector of the point $O$. Note that $\theta$ is a real number; $\theta=$ const. represents a space-time characteristic surface or wave front [1].

Now, as the continuity criterion guarantees that a boundary point can be considered as an interior point, the plane waves are employed in this paper to serve two purposes:

1. to demonstrate the physical effect of the continuity criterion: at such a boundary point, a plane wave, i.e., its amplitude and phase remains intact or undistorted across the boundary. Hence the non-reflecting property is justified as stated in $\S 2.1$;

2. to conduct phase error analysis at the boundary (in $\S 5.1$ ).

Figure $1 \mathrm{~b}$ sketches how a simple one-dimensional scalar plane wave propagates across a boundary $x=x_{0}$ in a plane $t=t_{0}$. The upper curve corresponds to $C^{1}$ continuity and exhibits no reflection. The lower curve corresponds to $C^{0}$ continuity with a possible kink of slope or phase error, spurious reflection may occur.

The $C^{1}$ continuity criterion is a generic NRBC. When incorporated in certain finite volume approaches with the hyperbolic conservation laws, a new class of absorbing NRBCs is created. As will be shown in $\S 3$ and $\S 4$, these NRBCs are simple but robust. The continuity criterion may also have direct applications, but that would be the topic of another NRBC paper.

\subsection{Relation of the continuity criterion to the characteristics-based NRBCs}

The continuity criterion is closely related to the well-known characteristics based NRBCs [6 - 9], although seemingly there is no apparent way to infer one from the other. First, both rely on the hyperbolicity of the equation system, which supports wave/disturbance propagation and characteristics. Secondly, for the characteristics-based NRBCs, as suggested in [6,7], for the incoming characteristic variable $w_{k}, \Delta w_{k}$ should be set to zero across the domain boundary. This step is consistent with the continuity criterion too. As such, their non-reflecting performance is expected to be similar.

However, the continuity criterion treats a multi-dimensional plane wave as a physical entity and keeps it intact or undistorted across the boundary surface to attain the 'non-reflecting' effect. This contrasts to the usual treatment of characteristic field decomposition in the characteristics-based NRBCs and is much simpler.

\section{The absorbing NRBC and the absorbing layer (matched layer)}

The continuity criterion is primarily applied to the absorbing NRBC. This absorbing NRBC is generally considered to be a difficult problem because the boundary conditions are subject to excessive restrictions. On the one hand, the given flow conditions must be satisfied; on the other hand, the boundary must be non-reflecting or absorbing for the outgoing waves. Conventionally, by determining the one-dimensional outgoing or incoming characteristics, the characteristics-based NRBCs modify the boundary conditions to an admissible one that handles the absorbing boundary problem. The process is termed 'hard absorbing' in $\S 1$ and becomes cumbersome when the flow is multidimensional and complicated. The perfectly matched layer or PML method [11, 12] offers an attractive treatment, in which a set of equations in the matched layer is prescribed and solved such that the outgoing wave is dissipated exponentially and absorbed. Such absorbing occurs in the matched layer and hence belongs to the 'soft absorbing'.

The new technique is shown to work for a large class of finite volume schemes that follow the two-stage procedure: an evolution (time-marching) stage and a reconstruction stage. The continuity criterion is here combined with the conservation laws in discretized form and yields a desirable absorbing NRBC - simple but robust. The non-reflecting property of the Type I absorbing NRBC is established in $\S 3.1$. Section 3.2 provides a general guideline for numerical implementation of the absorbing NRBC. The matched layer or absorbing layer is discussed in $\S 3.3$. 

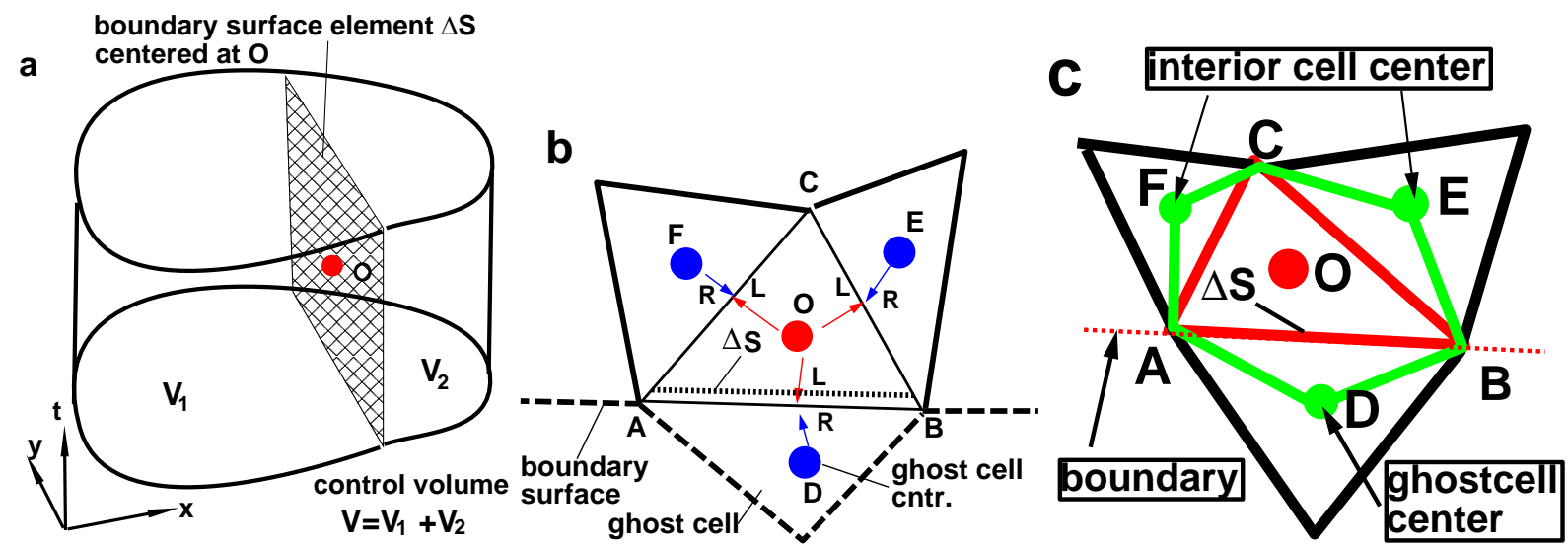

Figure 2. a: Boundary surface element $\Delta S$ as an internal interface of the control volume $V ; b$ and $c$ : Examples of 2-D numerical implementation: $b$ : A standard upwind scheme with an unstructured triangular grid; ' $R$ ' and ' $L$ ' represent respectively the right and left states for the Riemann solvers, they are obtained by extrapolated flow data from the current cell center $O$ and the neighboring cell centers $D, E$ and $F$. The prism cylinder based on $\triangle A B C$ is the space-time control volume. The boundary surface element $\triangle S$ practically coincides with the side $A B$. c: For the CE/SE method, with $A B$ as the boundary $\triangle S$, the space-time control volume $V$ is a hexagon cylinder based on $A D B E C F A$. In both cases, physical boundary conditions are given at the ghost cell center $D$.

\subsection{The absorbing NRBC}

After discretization, the boundary surface $S$ is also divided into small boundary elements, $\Delta S$, according to the grid. Let $V$ be a control volume which contains the boundary surface element $\Delta S$ as its internal interface (Fig. 2a). Note that $V$ may or may not be a grid cell. It will be shown that $\Delta S$ is non-reflective with any prescribed physical boundary conditions at the ghost cell center. No additional numerical implementation is needed. The mechanism works as follows: the $C^{1}$ continuity of the flow variables $\mathbf{V}$ across $\Delta S$ provides the non-reflecting property as shown in the next paragraph. Then the inconsistent flow data between the imposed boundary conditions and the outgoing wave/disturbance is treated as a 'numerical jump' around the boundary, captured, and absorbed by the shock-capturing scheme which is based on the hyperbolic conservation laws. As a result, a matched layer (ML) or absorbing layer is automatically generated around the boundary.

It is straightforward to prove the 'non-reflecting' property of the boundary surface element $\Delta S$. In a finite volume numerical approach, at the evolution stage (time-marching stage), for any prescribed physical boundary conditions at the ghost cell center, the integral conservation laws (2) are solved with the control volume $V$, yielding a spatial average $\mathbf{V}$ (or $\mathbf{U}$ ) over $V$ at the new time level $n+1$. At the reconstruction stage, by using a flux limiter, such as the minmod or the van Albada limiters etc., to recover the spatial gradients of $\mathbf{V}$ (i.e., $\mathbf{V}_{x}, \mathbf{V}_{y}$ and $\mathbf{V}_{z}$ ), this constant averaged $\mathbf{V}$ is rebuilt into $\mathbf{V}^{n+1}$ - a linear, high resolution (or second order accurate ) solution of (2) within the control volume $V$. It is the reconstruction stage that provides the non-reflecting property. Note that $\mathbf{V}^{n+1}$ represents a discretized solution of (2) or a discretized 'weak solution' of (4) within the entire control volume $V$ and that $\Delta S$ is an internal interface of $V$. As a linear vector function within $V, \mathbf{V}^{n+1}$ satisfies the $C^{1}$ continuity criterion at the surface center of $\Delta S$ (Fig. 2a), hence $\Delta S$ is non-reflective.

\subsection{Implementation of the absorbing boundary conditions}

Construction of the absorbing NRBC is straightforward too - no additional numerical implementation is required except the prescribed boundary conditions at the ghost cell centers, because an absorbing layer is automatically generated. For the class of finite volume approaches which contain the two-stage procedure of evolution (time-marching) and reconstruction, here are some general guidelines:

1. Choose an appropriate control volume, $V$, at the boundary and ensure that the boundary surface element, $\Delta S$, is an internal interface of $V$. For most of the finite volume schemes, usually, a control volume $V$ where the Gauss divergence theorem (2) is applied is equivalent to a grid cell. Then, $\Delta S$ may coincide with the cell surface at the 


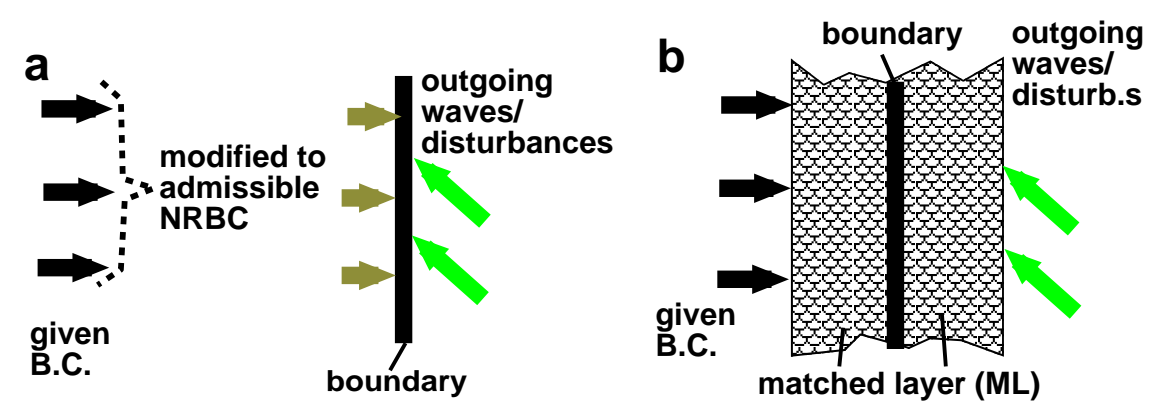

Figure 3. Comparison of absorbing NRBCs at an inlet boundary; a: Conventional 'hard' absorbing NRBC (characteristics-based); $b$ : Type I 'soft' absorbing NRBC, the matched layer plays the role as a buffer layer between the inconsistent flow data at domain boundary and interior.

domain boundary but is considered lying at the inner side of the boundary surface (Fig. 2b). For some schemes, e.g. the CE/SE scheme [14,15], a control volume $V$ is larger than a grid cell and $\Delta S$ is truly an internal interface of $V$ (Fig. 2c).

2. The finite volume approach follows the standard two-stage procedure: the evolution (time-marching) stage and the reconstruction stage by flux limiter. Nevertheless, even when the reconstruction stage is missing, the NRBC still holds, as the case is equivalent to a first order scheme, with all the spatial gradients vanished.

This absorbing NRBC is flexible and valid with practically any cell shapes, structured or unstructured. Figure $2 b$ and $2 \mathrm{c}$ show examples of the two-dimensional numerical implementation - the treatments in a standard upwind scheme and in the CE/SE method. Both will be used as numerical platforms for the computed examples. Flow data at the boundary cell center $O$ is updated based on flow data at its three neighboring cell centers $D, E$ and $F$. The divergence theorem is applied to the control volumes: the triangle cylinder $\triangle A B C$ or the hexagon cylinder $A D B E C F$. The side $A B$ is the non-reflecting boundary and an internal interface of the control volume as well.

\subsection{The matched layer (absorbing layer) near the boundary}

As the specified boundary conditions are imposed at the ghost cell centers, they also play a dual role of an absorbing (Type I) NRBC. Often, after a considerable long run-time, inconsistency between the imposed boundary conditions and the flow in the domain interior near the boundary may develop. Then, while the $C^{1}$ continuity across the boundary surface rules out spurious reflections, the shock-capturing scheme automatically captures the inconsistency in a 'matched layer' (ML) or 'absorbing layer'. Decaying of the inconsistency in the absorbing layer is fast. Depending on the required accuracy, the matched layer (ML) is typically only a few cells thick. It is non-physical, but plays an important role in separating and matching the inconsistent flow data at the boundary and the domain interior. The matching or absorbing is thus considered 'soft'.

The situation is similar to that in the PML (perfectly matched layer) method [11, 12], except that the 'matched layer' or absorbing layer is automatically generated and no special equations in the matched layer are needed.

\section{Numerical examples}

The absorbing NRBC is the primary but indirect application of the continuity criterion. As a matched layer or absorbing layer will be formed automatically, the absorbing NRBC is flexible and capable of handling complicated non-reflecting situations, such as with the 'difficult' cases where locally the flow disturbance may enter or exit the domain through the boundary and where the flow conditions could be supersonic along a portion of the boundary but subsonic along another portion of the same boundary.

Since computations in multi-dimensional space are of primary importance, in this section, the absorbing NRBC is tested in examples in two- or three-dimensional space with different finite volume schemes to demonstrate its performance and robustness. Here, a standard upwind scheme with an unstructured triangular grid, as depicted in Fig. 2b (for 2-D problems) and the space-time conservation element and solution element (CE/SE) method [13, 14] are chosen as the numerical platforms for computing the examples. The upwind scheme uses Roe's approximate Riemann solver and renders first order accuracy in time but high-resolution in space. The CE/SE scheme is nominally second order accurate in both space and time. 

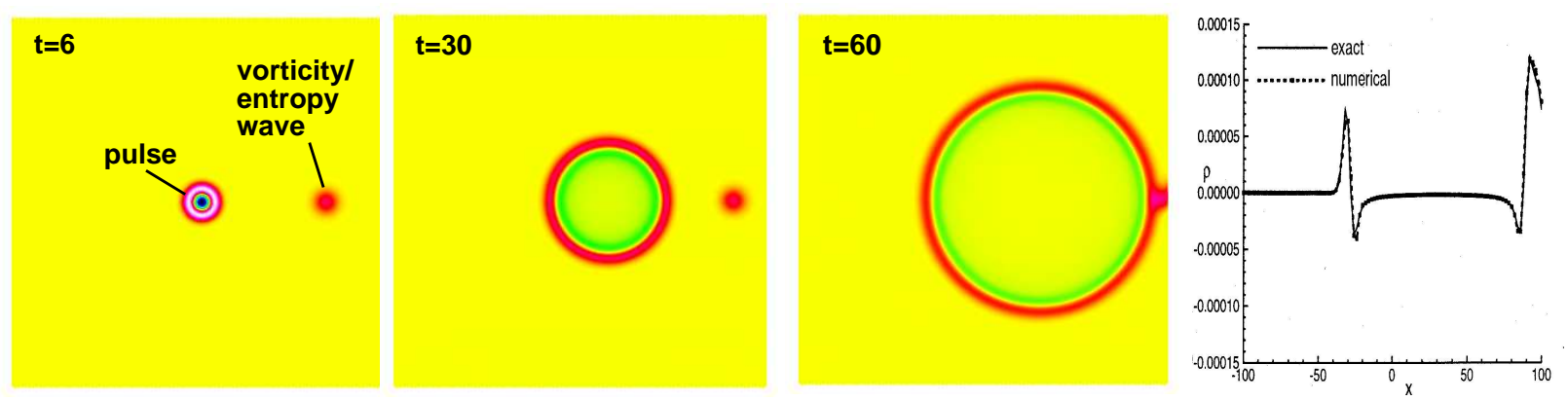

Figure 4. Propagation of a linear acoustic pulse with vorticity/entropy disturbances: density contours plots at $t=6,30,60$; with comparison to exact solution along the $x$ axis, showing no reflection at the outflow boundary. The CE/SE scheme is employed here.
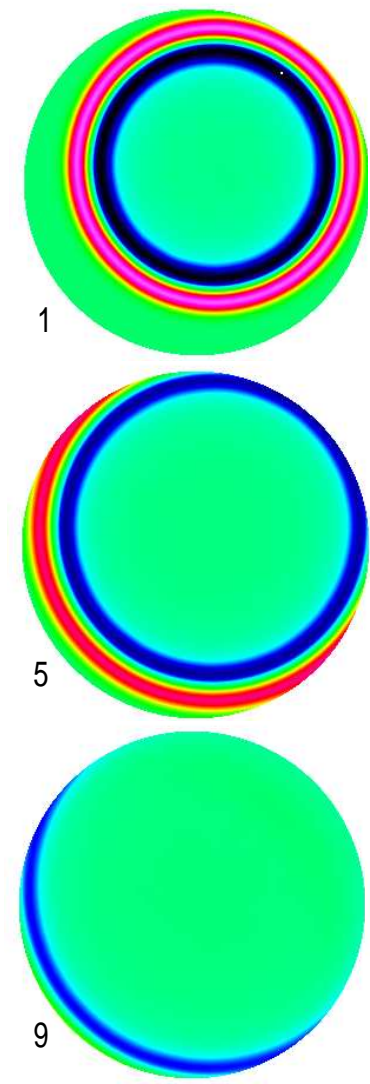
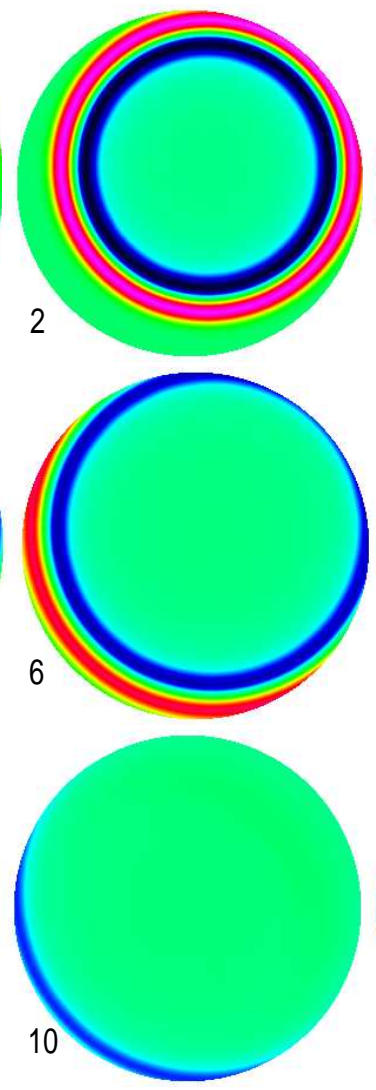
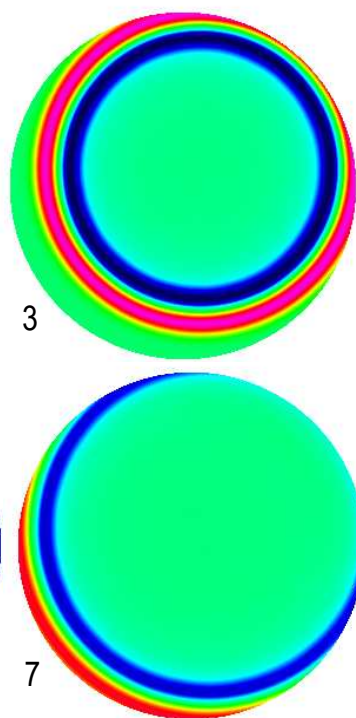

4
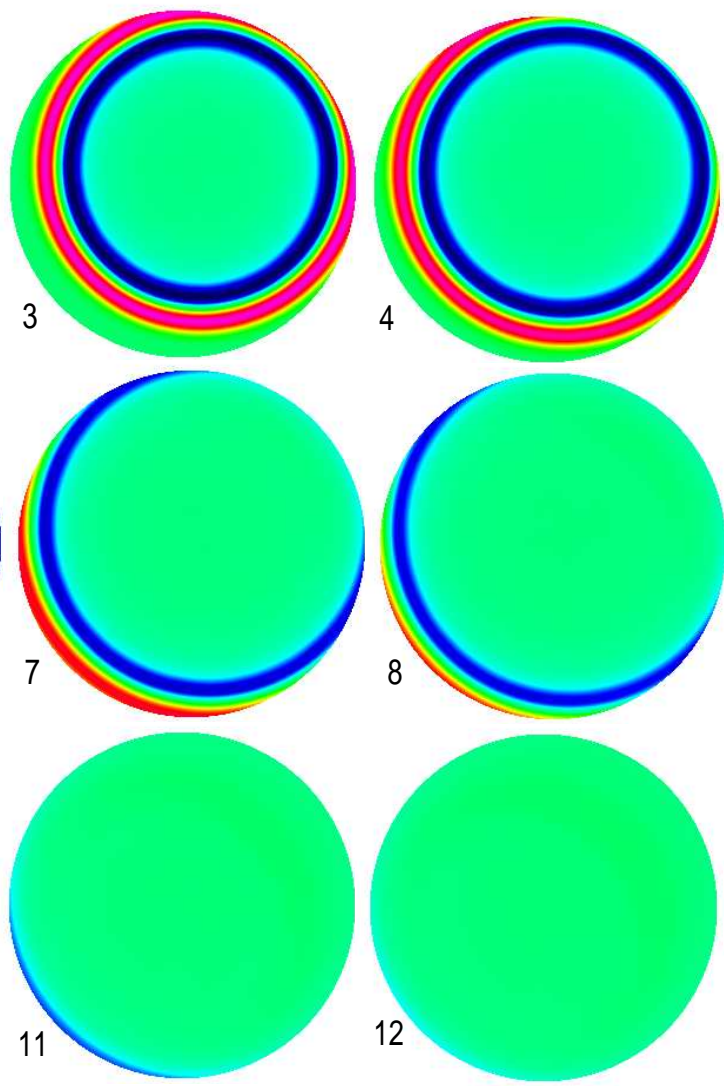

Figure 5. Plots 1-12, propagation of a Gaussian pulse in a circular domain from $t=60$ to $t=120$. The flow Mach number, $M$, is 0.2 at an angle of $45^{\circ}$. Although the flow is inclined and the boundary is curved, no visible reflection is observed. A first order Godunov upwind scheme is employed in this problem. The absorbing layer exists but probably too thin and weak to be visible.

\subsection{2-D acoustic pulse, entropy wave, and vorticity wave propagation}

This problem illustrates the propagation of the three basic types of linear waves in a two-dimensional domain [15, 16]. The computational domain in the $x-y$ plane is a square with $-100 \leqslant x \leqslant 100$, and $-100 \leqslant y \leqslant 100$. A uniform 

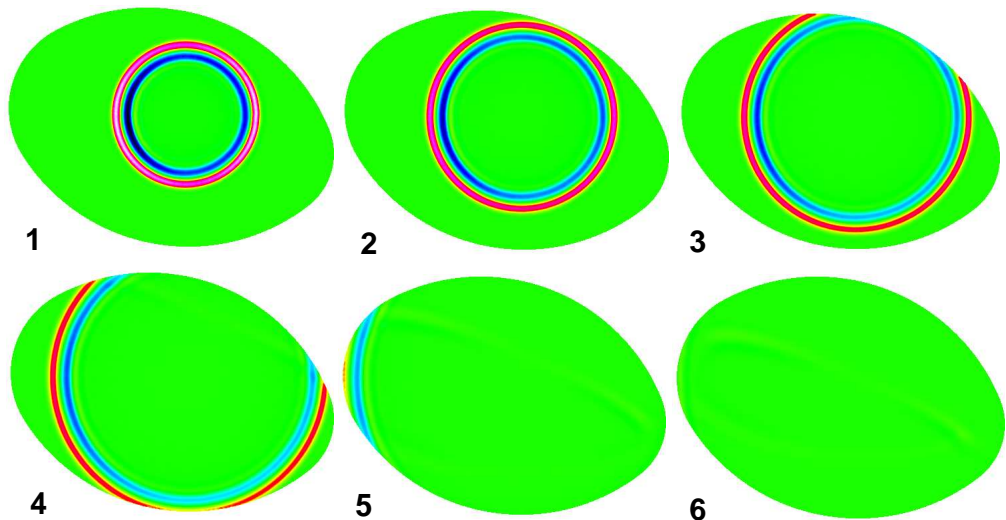

Figure 6. Plots 1-6, propagation of a Gaussian pulse in an egg-like domain at $t=150,200,250,300,350$, and 400. The flow Mach number, $M$, is 0.05 and directs at $45^{\circ}$. ( about 65,000 unstructured triangular cells, with the CE/SE scheme)

grid with 40, 000 uniform triangulated cells is used with the CE/SE scheme. Initially, a Gaussian acoustic pulse is located at the center of the domain $(0,0)$ and a weaker entropy/vorticity disturbance is located off the center at $(67,0)$, with a mean flow Mach number, $M=0.5$, in the $x$ direction. The corresponding non-dimensional pressure, density, and streamwise and transverse velocity components are given by

$$
\begin{gathered}
p=\frac{1}{\gamma}+\delta \mathrm{e}^{-\alpha_{1}\left(x^{2}+y^{2}\right)}, \quad \rho=1+\delta \mathrm{e}^{-\alpha_{1}\left(x^{2}+y^{2}\right)}+0.1 \delta \mathrm{e}^{-\alpha_{2}\left[(x-67)^{2}+y^{2}\right]} \\
u=M+0.04 \delta y \mathrm{e}^{-\alpha_{2}\left[(x-67)^{2}+y^{2}\right]}, \quad v=-0.04 \delta(x-67) \mathrm{e}^{-\alpha_{2}\left[(x-67)^{2}+y^{2}\right]}
\end{gathered}
$$

where $\alpha_{1}=\ln 2 / 9, \alpha_{2}=\ln 2 / 25$, and with a small amplitude factor, $\delta=0.001$, the two-dimensional Euler equations are practically linearized. At all four boundaries, flow variables are given with the $M=0.5$ mean flow conditions:

$$
p_{a}=\frac{1}{\gamma}, \rho=1, u=M=0.5, v=0 .
$$

These conditions play the role as an absorbing NRBC. Figure 4 illustrates the evolution of the density contours at different time steps with comparison to the exact solutions (perturbation part) along the $x$ axis. It is observed that the numerical solutions agree well with the exact solutions and that there is no reflection along $x$ axis. As the the waves are weak, the absorbing layer is not visible.

\subsection{2-D acoustic pulse propagation}

The second example is a two-dimensional subsonic non-linear wave propagation problem. A circular computational domain in the $x-y$ plane is used, ranging from $-100 \leqslant x \leqslant 100$, and $-100 \leqslant y \leqslant 100$ (Fig. 5). There are about 87,200 unstructured triangular cells in the domain, and the two-dimensional unsteady Euler equation system is solved by a simple first order accurate Godunov upwind scheme. Initially, a Gaussian acoustic pulse is located at (10,10) in the domain, with a mean flow Mach number, $M$, of 0.2 in a direction $45^{\circ}$ inclined to the $x$ axis.

$$
p=e^{-\ln 2\left(x^{2}+y^{2}\right) / 9}+1 / 1.4, \quad \rho=e^{-\ln 2\left(x^{2}+y^{2}\right) / 9}+1, u=v=0.1 \sqrt{2} .
$$

The Type I NRBC which is identical to the initial conditions i.e. $p=1 / 1.4, \rho=1, u=v=0.1 \sqrt{2}$, is imposed at the circular boundary. As time elapses, the non-linear pulse convects with the flow and propagates. Figure 5 shows twelve frames of the isobars from $t=65$ to $t=120$ at an increment of $\Delta t=5$. When the pulse propagates towards the boundary and exits the domain, no visible reflection is observed. The matched layer is too thin to be visualized. The curved boundary and the oblique flow direction help to demonstrate the capability of the absorbing NRBC. 

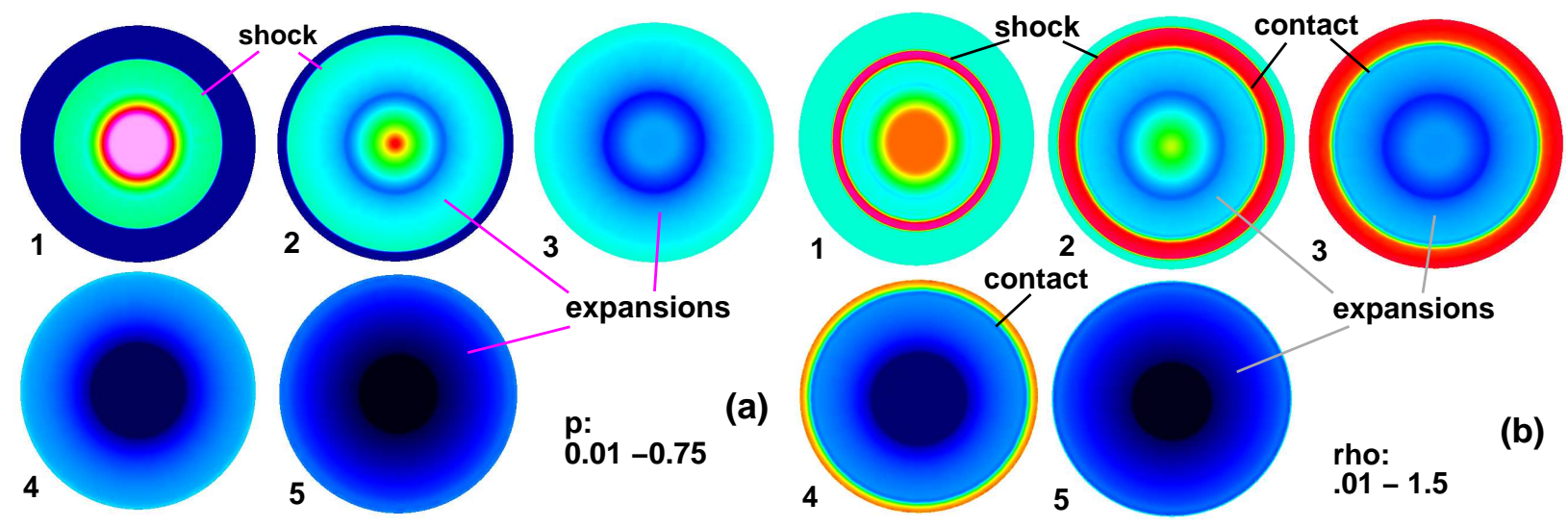

Figure 7. Two-dimensional radial Riemann problem, showing how a shock and a contact discontinuity are perfectly absorbed by the boundary. Plot 1-5 correspond to their propagation at $t=50,100,125,175$, and 225 . The mean flow is stationary $(M=0)$.

\subsection{2-D acoustic pulse propagation in an egg-like domain}

This example demonstrates that the absorbing NRBC can be applied to a domain of more general shape. All the conditions are similar to the previous example except that the domain has the shape of an egg and that the mean flow Mach number, $M$, is 0.05 . The numerical scheme used here is the CE/SE scheme. Figure 6 shows six frames of the isobars from $t=150$ to $t=400$ at an increment of $\Delta t=50$. In this case, when the pulse propagates towards the boundary and exits the domain, the absorbing NRBC performs generally well, except some minor reflection in the last 3 plots, due to certain phase errors that will be described in $\S 5.1$.

\subsection{Propagation of discontinuous waves (1)}

Here the problem is a two-dimensional radial Riemann problem and the physical phenomena are complicated. This example demonstrates with the CE/SE scheme how a shock and a contact discontinuity are absorbed by the boundary. The same circular domain as in $\S 4.2$ is employed. The mean flow is stationary: $\left(\rho_{0}, u_{0}, v_{0}, p_{0}\right)=$ $(0.5,0,0,0.1 / 1.4)$, which are also imposed at the ghost cell centers at the circular boundary as the absorbing NRBC. Initially, within an inner circle of radius $r=50$, a state of high pressure and density is given: $(\rho, u, v, p)=$ $(1.0,0,0,1.0 / 1.4)$. Figure 7 shows five plots of isobars and isopycnics (density contours) at different time steps. In Fig. 7a, the first two plots show how the circular shock approaches the absorbing boundary. From the third plot on, the shock passes through the boundary and is absorbed by the boundary, leaving no trace of reflection. Figure $7 \mathrm{~b}$ not only demonstrates how the shock leaves the domain, but also the evolution of the contact discontinuity. In the fifth plot, the contact already passes through the boundary and no reflection appears.

\subsection{Propagation of discontinuous waves (2)}

This radial Riemann problem is similar to the previous example, except that the mean and core flows move at $M=$ 0.2 and $45^{\circ}$. Initially, the mean flow and core flow data are respectively $\left(\rho_{0}, u_{0}, v_{0}, p_{0}\right)=(0.5,0.1414,0.1414,0.1 / 1.4)$, and $\left(\rho_{i}, u_{i}, v_{i}, p_{i}\right)=(1.0,0.1414,0.1414,1.0 / 1.4)$. Figure 8 shows eight plots of isopycnics (density contours) at different time steps. The circle-like shock moves towards the northeast direction in plots 1 and 2 . In plots 3 and 4 , the shock is almost fully absorbed by the boundary, leaving no visible reflections. From plot 5 on, the contact propagates slowly towards the boundary and is well absorbed. A high-resolution upwind scheme with a minmod flux limiter is employed for computing this case.

\subsection{2-D subsonic cavity flow}

Figure 9 illustrates the instantaneous isobars for a cavity noise problem. The grid consists of 42, 000 triangulated structured cells. The problem is a $M=0.8$ flow past a rectangular cavity of aspect ratio of 6.5 . At the cavity walls, no slip boundary condition is imposed. Due to vortex shedding and acoustic feedback at the cavity edges, strong nonlinear acoustic waves are generated and propagate in both upstream and downstream directions [17]. Figure 10 


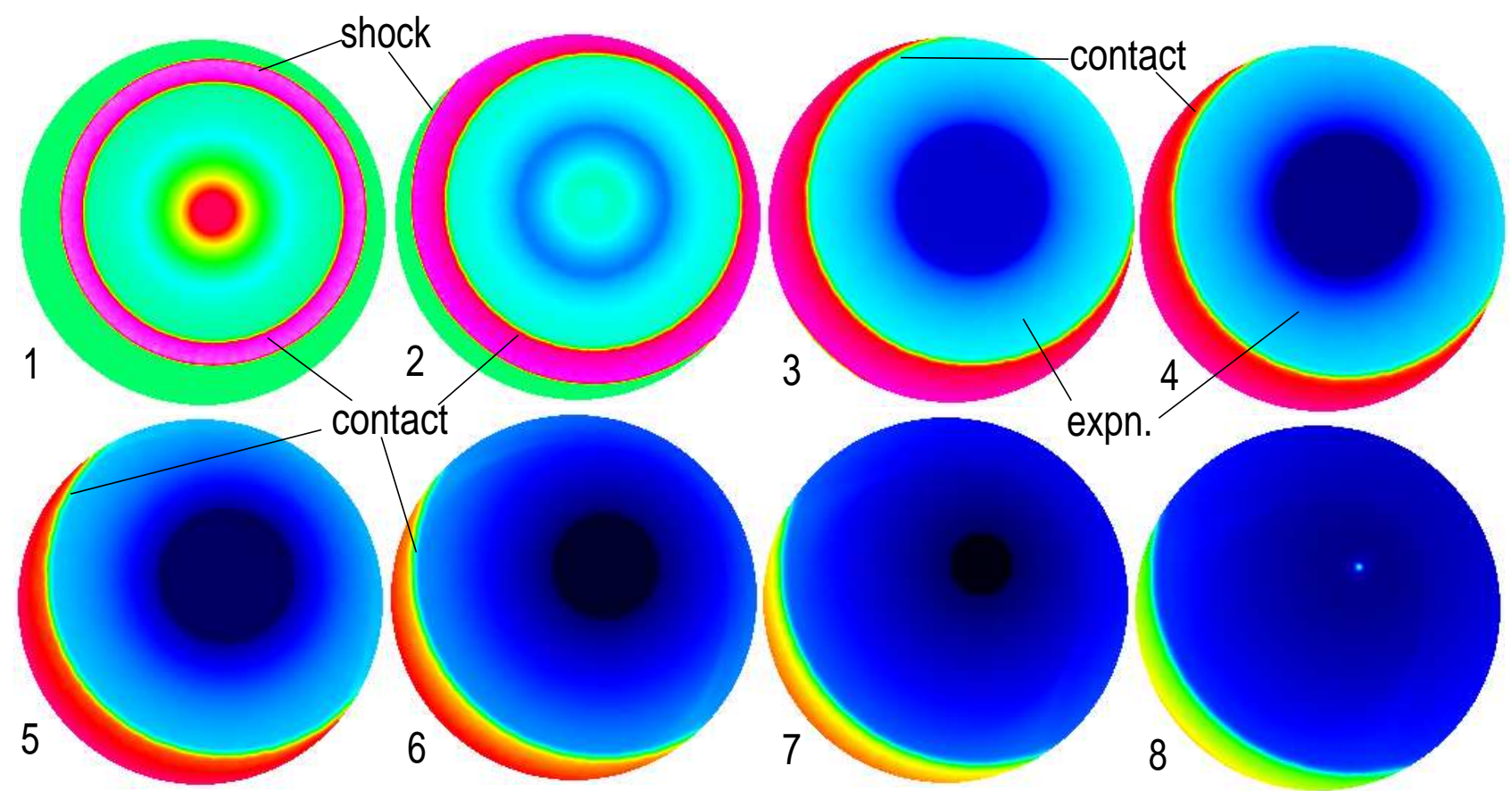

Figure 8. Two-dimensional Riemann problem with mean and core flows moving at $45^{O}$ and $M=0.2$, showing how the the shock and contact discontinuity are absorbed at the boundary. Plots 1-8 correspond to the density contours at $t=40,60,80,90,100,120,140$, and 160 .

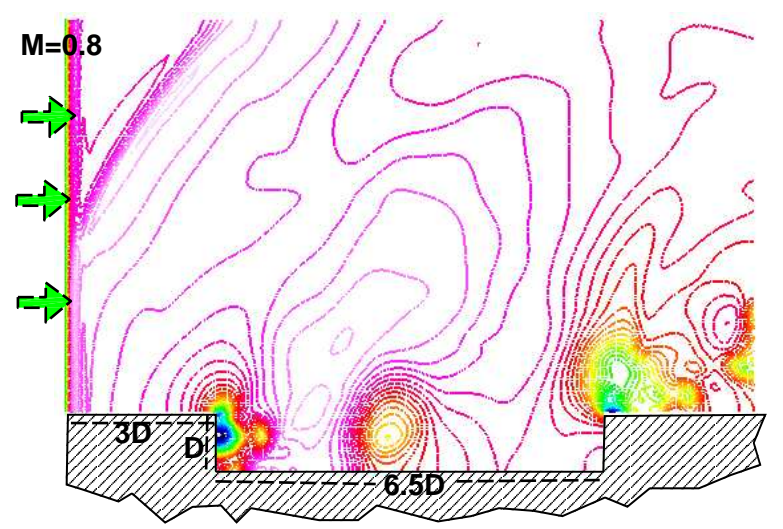

Figure 9. Isobars snapshot for a cavity noise problem (Mach number $M=0.8$ ), showing inflow NRBC and its absorbing property. Also, no visible reflection is found at the top and the outflow boundary

is an enlargement of Fig. 9 around the inflow area, revealing the details of the contours at the matched layer. It is observed that there is no spurious reflection and that the matched layer (absorbing layer) is about 4-5 cells thick. A $\mathrm{CE} / \mathrm{SE}$ type Navier-Stokes solver is used in the computation.

\subsection{3-D circular jet screech problem}

The absorbing NRBC can be extended to three-dimensional space problems in a consistent way. Figure 11 is the sketch of an underexpanded circular jet in three-dimensional space. The jet nozzle protrudes into the computational domain by $S=2 D, D$ being the inner diameter of the nozzle. The unstructured mesh contains 3.7 million hexahedral cells. At the inlet plane, an ambient (stationary) condition or the Type I absorbing NRBC is specified. Jet flow at higher pressure (with jet Mach number $M_{j}=1.42$ ) is specified at the nozzle exit. For over or under expanded jets, 

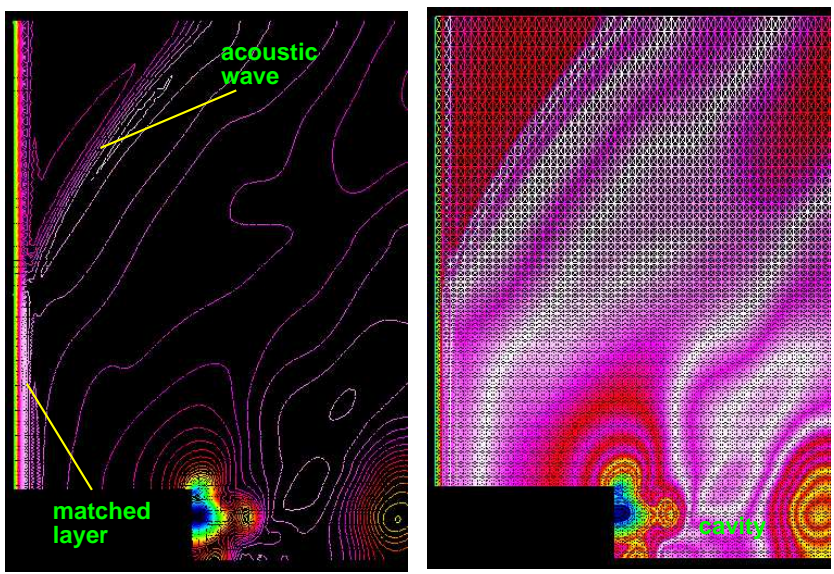

Figure 10. Details of the contours at the inflow boundary, showing the matched layer (absorbing layer) at the inlet and its spreading over the grid.
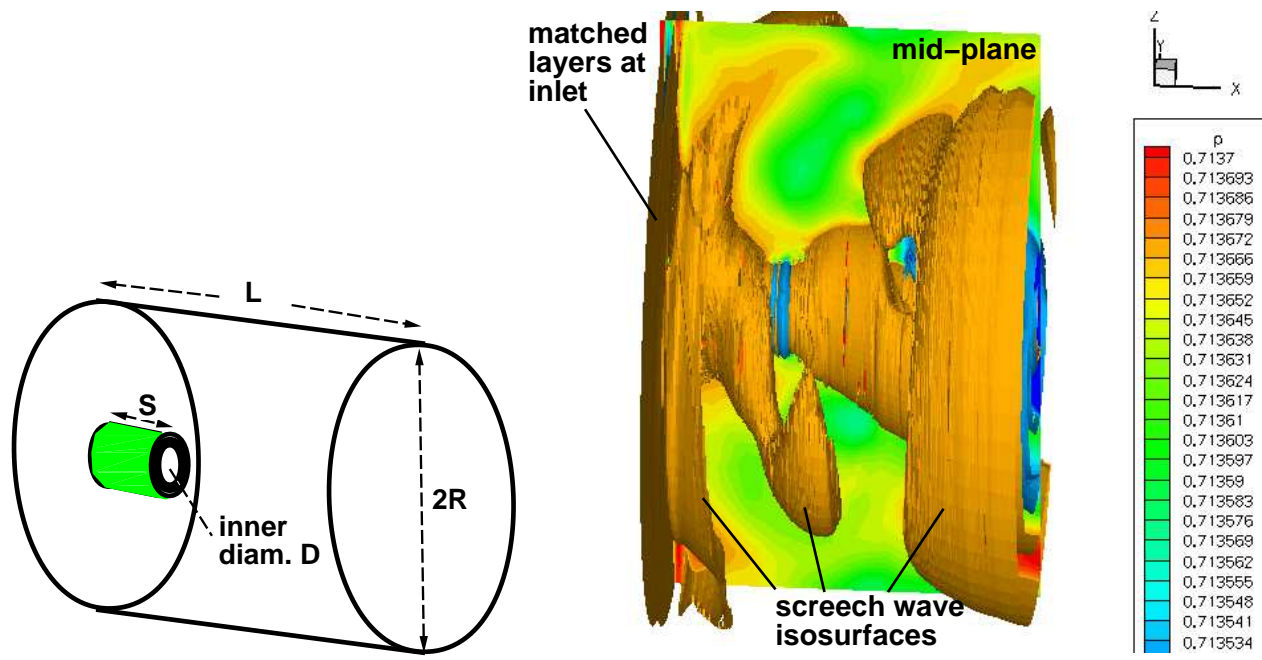

Figure 11. Left: Sketch of the 3-D circular jet screech problem and computational domain, jet Mach number $M_{j}=1.42, S=2 D, L=10 D$ and $R=5.5 D$. Right: instantaneous pressure isosurfaces, showing screech waves, and in particular, the disc of matched layer at the ambient inlet.

screech tone noise and waves may occur in a feedback loop in which the instability waves (vortices) in the shear layer interact with the shock-cell structure in the jet core and generate screech waves that propagate upstream to the nozzle lip triggering new vortices. A CE/SE type three-dimensional Navier-Stokes solver is employed for the work. Figure 11 also shows a snapshot of the pressure isosurfaces after running 510,000 time steps. The helical screech waves are clearly illustrated. At the inlet, an absorbing layer (or ML) is automatically formed as shown in Fig. 11.

\section{Discussions on the NRBCs}

Generally, the new NRBC exhibits little reflection. However, due to discretization errors and the possible consequent phase error at the physical wave front, there are situations when the NRBC may fail and discernible reflections occur. Limitations and remedies are briefly discussed here.

\subsection{Limitation of the NRBCs}

Due to discretization and the possible consequent phase error, the accuracy of the NRBC could be degraded. Consider the Fourier mode in a plane wave solution (5), i.e., $e^{i(\mathbf{k} \bullet \mathbf{x}-\omega t)}$. Here, $\theta(\mathbf{x}, t)=\mathbf{k} \bullet \mathbf{x}-\omega t$ is the phase of 


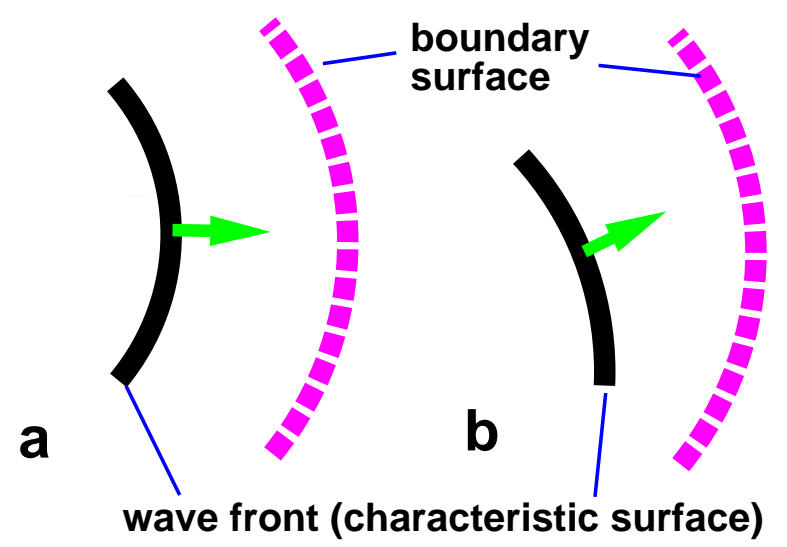

Figure 12. Wave propagation across a boundary; a: wave front coincides with boundary surface, best non-reflecting effect is obtained; $b$ : wave front is inclined to boundary surface, phase error may occur and cause spurious reflection. Arrows indicate wave propagation direction (direction of wave number vector)
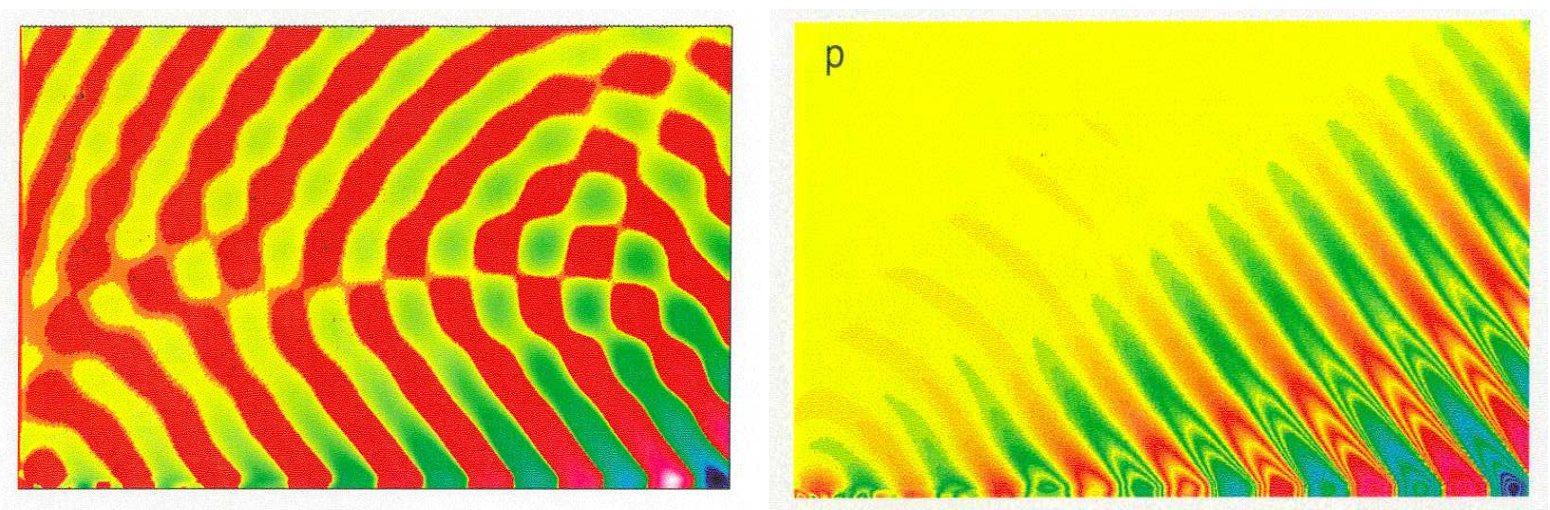

Figure 13. Mach radiation from a $M=2$ axisymmetric jet; Top: no buffer zone is added, flow field is contaminated; Bottom: with buffer zone added (not shown), showing a clean acoustic field.

the wave mode, with $\mathbf{k}$ being the given wave number vector in the propagation direction, and $\omega$ the frequency. The characteristic surface, $\theta(\mathbf{x}, t)=$ const., stands for a wavefront ( see e.g., Courant and Hilbert [1] or Hirsch [8]). After discretization, the center, $O$, of $\Delta S$ (Fig. 2a) is employed to represent the entire $\Delta S$ (a hyper-surface in both time and space), both spatial and temporal phase errors may occur. While the temporal phase error is part of the discretization error, the spatial phase error is closely related to the spurious reflections and is less tolerable. Assume that time $t$ is held unchanged, the spatial phase error can be easily analysed: Let $\mathbf{x}=(x, y, z)$ be the position vector of any point on $\Delta S$ and $\mathbf{x}_{O}$ the position vector of the center $O$. Then, the spatial phase error $\Delta \theta$ due to replacing $\mathbf{x}$ by $\mathbf{x}_{O}$ is:

$$
\Delta \theta=\mathbf{k} \bullet \mathbf{x}-\mathbf{k} \bullet \mathbf{x}_{O}=\mathbf{k} \bullet\left(\mathbf{x}-\mathbf{x}_{O}\right)
$$

note that the vector $\Delta \mathrm{x}=\mathrm{x}-\mathrm{x}_{O}$ lies on $\Delta S$, and $\Delta \theta=0$ when $\mathbf{k}$ is normal to $\Delta S$. Therefore, spurious reflection is minimized when the wave front (characteristic surface) coincides with the boundary (Fig. 12a). Otherwise, a phase error of order $O(\Delta \mathbf{x})$ may be introduced and cause numerical reflection. It should be noted that the above analysis involves only discretization and is hence valid for other NRBC techniques such as the characteristics-based NRBC.

It is conceivable that changing the orientation of the boundary will help to reduce spurious reflections. But in practice, an effective remedy is to impose a buffer/sponge zone between the boundary and the domain interior. Although the same governing equations (1) or (2) are employed in the sponge zone, numerical damping is highly increased to diminish the wave/disturbance amplitude before it reaches the boundary and to minimize the spurious reflection typically by grid stretching. An example is illustrated here to demonstrate the effectiveness of the buffer/sponge zone. 


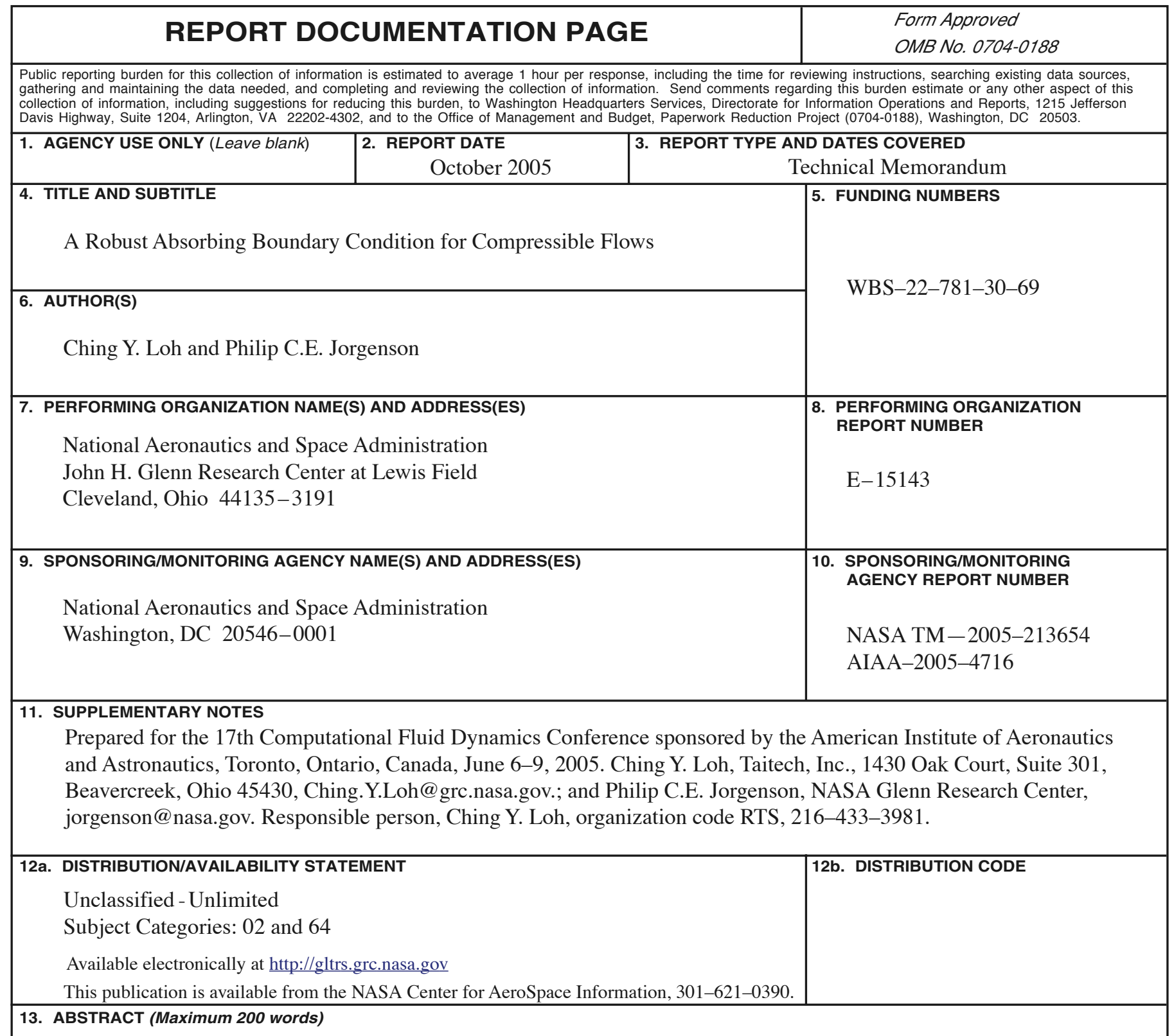

An absorbing non-reflecting boundary condition (NRBC) for practical computations in fluid dynamics and aeroacoustics is presented with theoretical proof. This paper is a continuation and improvement of a previous paper by the author. The absorbing NRBC technique is based on a first principle of non-reflecting, which contains the essential physics that a plane wave solution (Fourier mode) of the Euler equations remains intact across the boundary. When combined with the hyperbolic conservation laws, the NRBC is simple and robust, provided the numerical scheme maintains locally a solution of second order (or higher) accuracy near the boundary. Several numerical examples in multi-dimensional spaces are illustrated to demonstrate its robustness in practical computations.

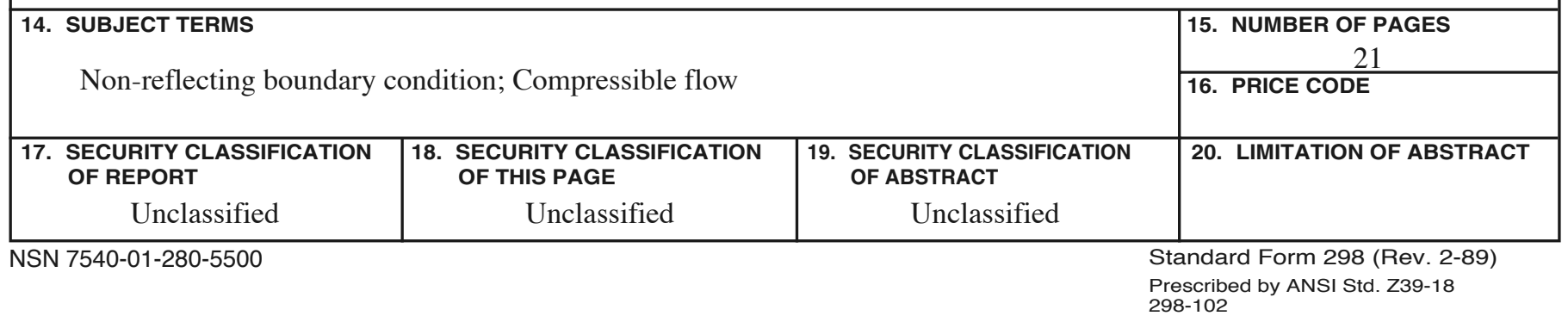



